\title{
Analysis of Muscle Fatigue Using Electromyography Signals in Gastrocnemius Muscle during Isometric Plantar Flexion
}

\author{
Mayank Patel ${ }^{1 *}$, Navaneethkrishna Makaram², Sriram Balasubramanian¹, Swaminathan \\ Ramakrishnan $^{2}$ \\ ${ }^{1}$ School of Biomedical Engineering, Drexel University, Philadelphia, Pennsylvania, United States of America. \\ 2 Department of Applied Mechanics, NIID Lab, IIT Madras, Chennai, India. \\ * Corresponding author. Tel.: +1 215-237-1584; email: mjp438@drexel.edu \\ Manuscript submitted August 20, 2017; accepted December 12, 2017. \\ doi: 10.17706/ijbbb.2018.8.2.100-106
}

\begin{abstract}
Fatigue during plantar flexion and the gastrocnemius muscles causes an increase in mediolateral sway and postural control impairment. The purpose of this study is to analyze the variation in the surface electromyography (sEMG) characteristics during isometric plantar flexion. For this, sEMG signals are recorded from the medial and lateral gastrocnemius muscle from 8 subjects and a minimum of 3 trials each, leading to a total of 25 trials per leg. The subjects performed isometric plantar flexion and are asked to hold the position until fatigue. The result shows that the endurance time of each subject vary. On comparing the endurance between legs, a marginal difference is observed. The recorded signal is nonstationary in nature. On visual inspection, the signals show an increase in amplitude with fatigue. The features such as RMS show an increase in value with $20.31 \%$ in right leg and $13.93 \%$ in the left leg for the signals from medial and lateral gastrocnemius. The proposed muscle fatigue index is a co-contraction based feature and provides better separation between non-fatigue and fatigue. The P-values obtained indicate that the proposed feature performs better than the conventional RMS value with a significance level of $P<0.01$. This study can be extended to analyze other neuromuscular conditions.
\end{abstract}

Key words: Gastrocnemius, fatigue, root mean square value, surface electromyography, plantar flexion.

\section{Introduction}

Fatigue in the muscles occurs when the cell lacks the energy to continue its activation [1]. Muscle fatigue can be defined as the inability to provide a certain amount of force required to complete a task [2]. Fatigue can also occur due to Parkinson's disease, endocrine disturbances, carcinoma, malnutrition, and immobilization [3].

In neuromuscular diseases, electrical simulation can often occur in muscle fibers, causing fatigue without any significant activity [4]. The analysis and study of fatigue is crucial to the understanding of the human body because fatigue in one location can cause affects in other parts of the body [5].

In the analysis of fatigue, many approaches have come about in the recent years. Isometric strength tests, exercise endurance test, muscle biopsy, muscle imaging, and surface electromyography (sEMG) are common methods [3].

In this study sEMG is used to analyze muscle fatigue. sEMG represents the electrical signals that results 
from contraction from muscles [6]. These contain information regarding the motor unit action potential trains that come from the motor units in the muscle [7].

There are multiple factors in which SEMG signals vary between non-fatigue and fatigue conditions [8]. The properties signals change because the nerves decrease the firing of the high-frequency motor unit action potentials [9]. They also change in amplitude of the signal due to chemical imbalances caused by the loss of energy in the muscle cells [6].

The gastrocnemius is a crucial muscle in the body as its function is to flex the foot and is constantly being used in everyday situations for even the simplest tasks like walking [5]. Fatigue in the gastrocnemius results in impairment in the balance of the body. During planar flexion, it is observed that an increase in mediolateral sway occurs and results in postural control impairment.

sEMG features such as the Root Mean Square (RMS) value is considered as one of the key component of analysis. It illustrates the activity in the muscle, and is affected by the firing rate of the cells, motor unit potential, unit discharge, and number of active muscle cells [10].

In this work, an attempt is made to analyze the variations in SEMG signal characteristics before and after fatigue in lower limb muscles during isometric plantar flexion exercise.

\section{Methodology}

\subsection{Experimental Protocol}

Eight male subjects are participants in the study with the range of ages being 19-32. A minimum of three trials is taken for each subject, giving a total number of trials of 25. Trials on the same subject are not done within 24 hours of each other to guarantee sufficient time for the muscles to recover. The subjects are not physically trained. Further, they were informed about the experiment and signed a consent form based on the Helsinki Declaration.

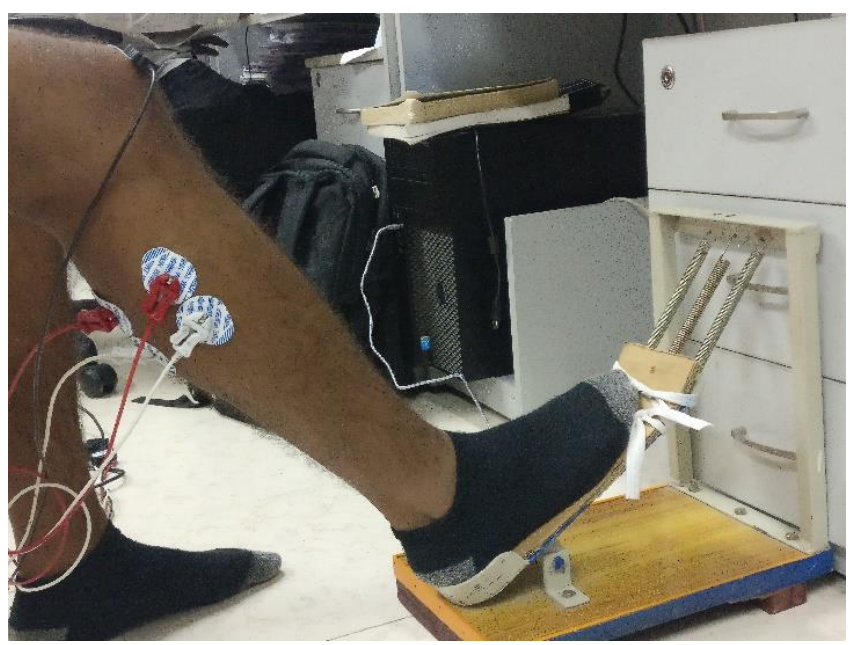

Fig. 1. Setup of experiment is shown. Electrodes are placed on the heads of the gastrocnemius muscles. The resisting force derives from the three springs.

Subjects are seated in a stationary arm chair. After being seated, participants are instructed to maintain their torso at 90 degrees with their femur bone. The knee is bent such that an angle of 120-degree is made between the tibia and the femur.

The participant's foot lies upon the following apparatus as illustrated by Fig. 1. Having the ankle placed on the apparatus, a 90-degree angle should be made between the base of the foot and the tibia. The subject is asked to perform plantar flexion exercise to apply force on the platform, lowering the foot angle by 11 
degrees. This displacement is equivalent to an application of a force of $43.1 \mathrm{~N}$. The participants are asked to maintain this angle until a drop of two degrees is observed, indicating fatigue. The angle is measured using an accelerometer sensor. During the experiment, verbal encouragement is constantly given.

$\mathrm{Ag} / \mathrm{AgCl}$ surface electrodes are placed upon both the lateral and medial gastrocnemius heads with the patella as the reference electrode. The interelectrode distance is 2 centimeters and are placed longitudinally over the muscles. The EMG signals are sampled at $10 \mathrm{kHz}$, and initially bandpass filtered from $5 \mathrm{~Hz}$ to 1000 Hz. The data acquisition system used is Biopac MP36 [5]. This experiment is performed on both the left and right leg separately.

\subsection{Processing}

The acquired signals are further analyzed using MATLAB software. The files are separated, organized, and filtered again from $5 \mathrm{~Hz}$ to $400 \mathrm{~Hz}$. A $50 \mathrm{~Hz}$ notch filter is used to remove the power line artifacts. The start and stop time of the experiment is noted. The initial and final window $1000 \mathrm{~ms}$ of the signal are used for analysis.

\subsection{Root Mean Square}

The RMS is a time domain feature that gives insight into the amplitude by providing a measure of power of the signal. The equation is given by:

$$
R M S=\sqrt{\frac{1}{N} \sum_{i=1}^{N} x_{i}^{2}}
$$

where $x_{i}$ is the voltage value of the signal at $i^{\text {th }}$ instant and $\mathrm{N}$ is the total number of points being analyzed.

\subsection{Muscle Fatigue Index}

The muscle fatigue index is a proposed co-contraction based feature and is given by:

$$
\text { Muscle Fatigue Index }=\sqrt{R M S_{\text {medial }}^{2}+R M S_{\text {lateral }}^{2}}
$$

where $R M S_{\text {medial }}$ is the RMS value from the medial gastrocnemius and $R M S_{\text {lateral }}$ is the RMS value from the lateral gastrocnemius.

\section{Results and Discussion}

There is a total of 25 trials in each leg from 8 subjects conducted in this experiment. Fig. 2 illustrates the representative sEMG signals. In Fig. 2a, the signal from the lateral gastrocnemius of the left leg is shown. It is seen that the amplitude ranges between -1.0 and $1.0 \mathrm{mV}$ and the amplitude values increase with time. The signal from the medial gastrocnemius of the left leg is shown in Fig. $2 \mathrm{~b}$. A similar trend is seen, but the amplitude ranges from -0.5 to $0.5 \mathrm{mV}$. Fig. $2 \mathrm{c}$ and $2 \mathrm{~d}$ shows the lateral and medial gastrocnemius signal of the right leg respectively. It is seen that both the muscles have similar amplitude ranges and increasing trend is also observed with fatigue.

Comprehensive analysis of the endurance across subjects is shown in Fig. 3. Based on the scatter plot, it is seen that nearly 13 out of 25 trials have right leg with longer endurance time. On average, the right can perform for $7.29 \%$ longer than the left leg.

In this experiment, the first and last $1000 \mathrm{~ms}$ of sEMG signals are considered as non-fatigue and fatigue conditions, respectively. Fig. 4 represents the segmented signals acquired from the left leg that is shown in 
Fig. 2a and 2b. In Fig. 4, an increase in amplitude is seen from non-fatigue to fatigue case. In fatigue, a synchronization of motor units occurs and this increase in amplitude may indicate this phenomenon [3]. Further, there is more scatter in the case of fatigue. A higher standard deviation is observed in the case of lateral pre- and post-fatigue.

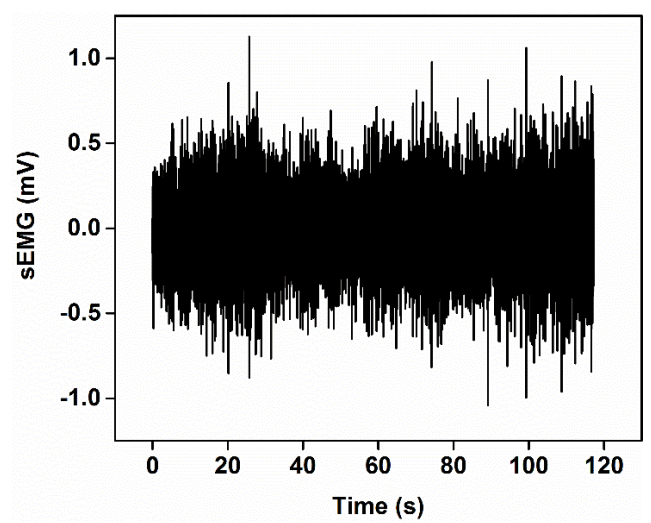

(a) Left lateral

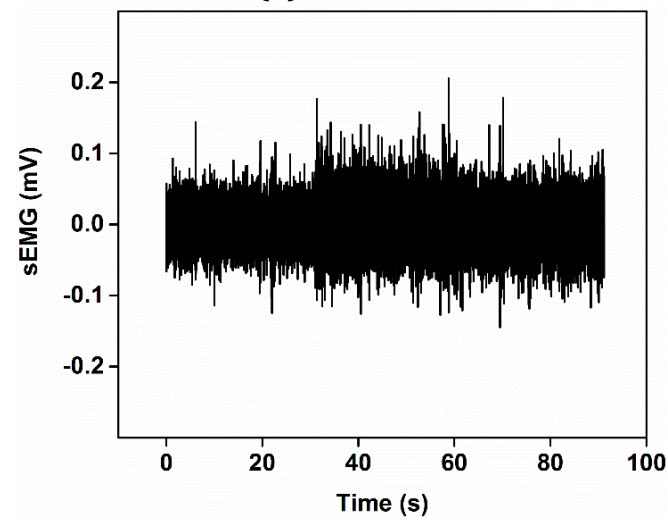

(c) Right lateral

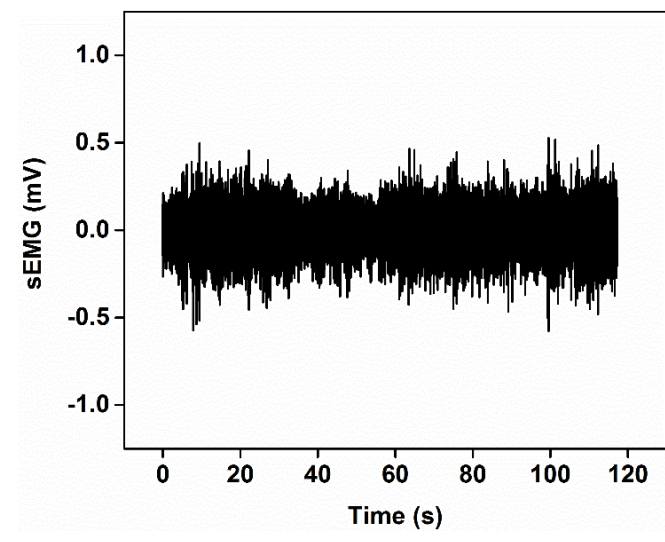

(b) Left medial

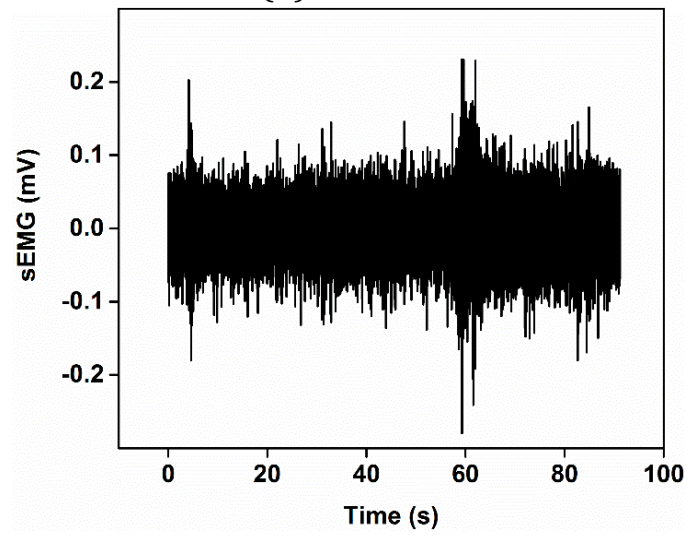

(d) Right medial

Fig 2. The full sEMG signal of a single trial is shown. The signal from the two heads of the gastrocnemius on the left leg are shown above and the signal from the two heads on the right leg are shown below. Amplitude increases as the experiment progresses in all cases.

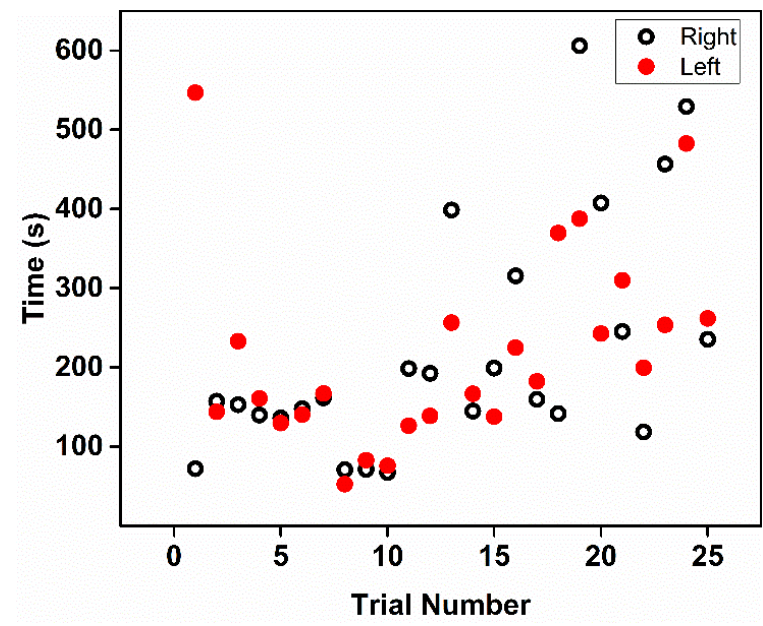

Fig. 3. The scatterplot of the length of time between the right leg and the left leg for the same trials is shown above. There was no clear dominant leg with 13 trials having a longer endurance time in the right leg and 12 in the left. 
In Table 1, the difference between the RMS values in non-fatigue and fatigue is shown as a percent increase. The table indicates that the right leg had an RMS percent increase of 1.5 times that of the left leg. There is a marginal difference between the lateral and medial gastrocnemius RMS increase.

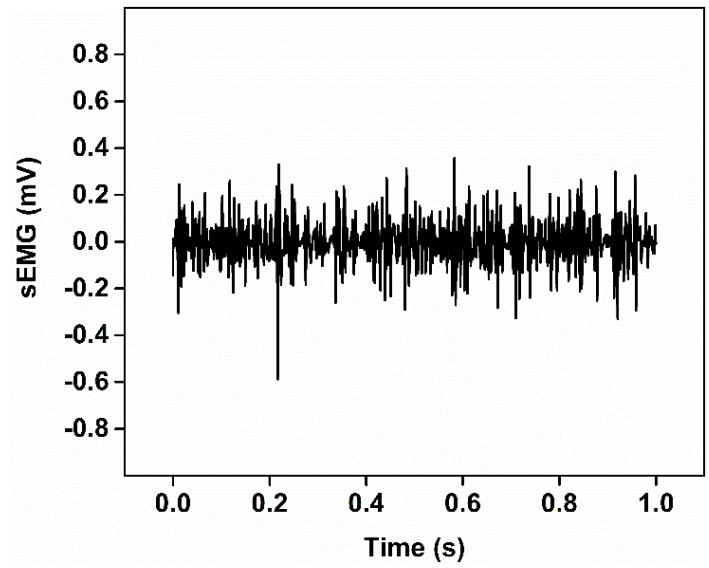

(a) Lateral non-fatigue

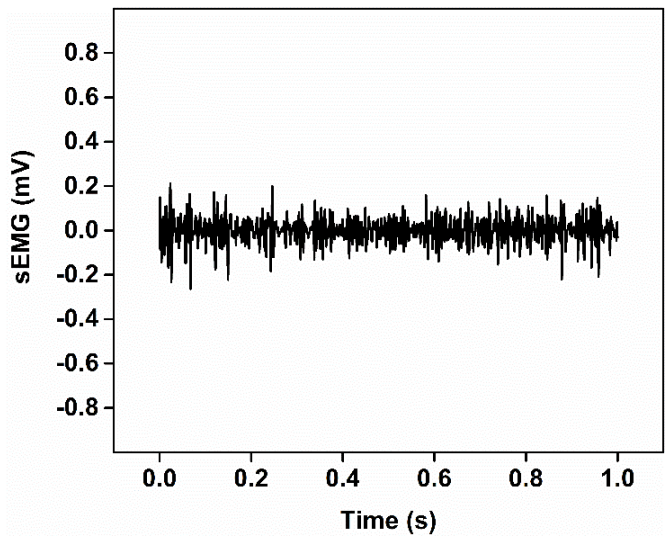

(c) Medial non-fatigue

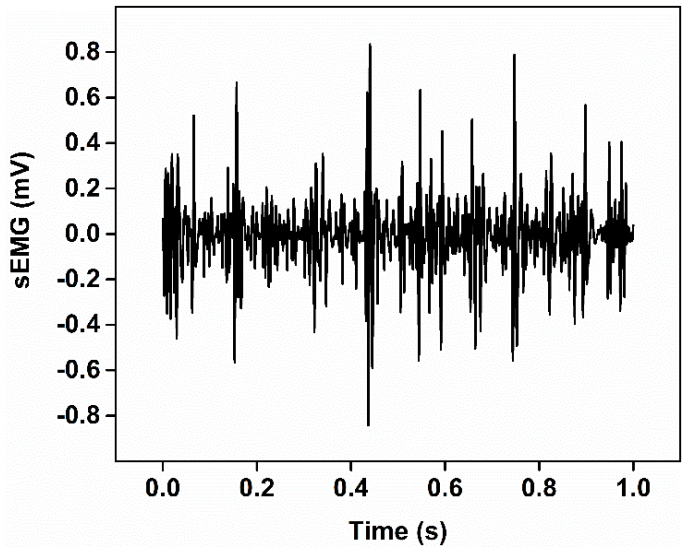

(b) Lateral fatgiue

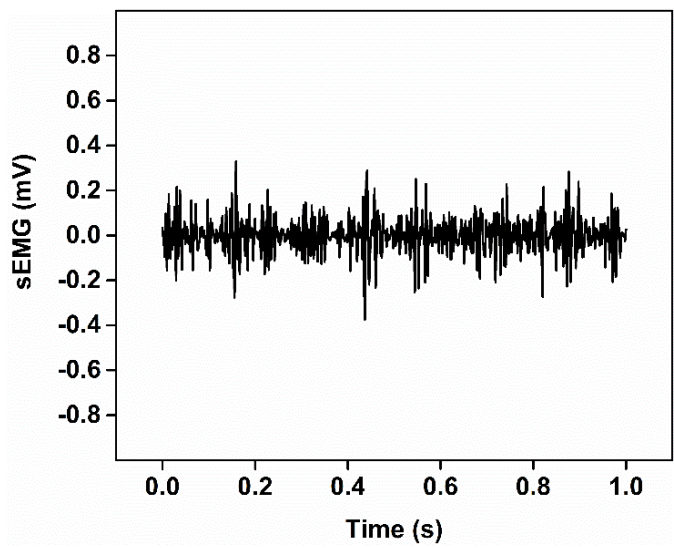

(d) Medial fatigue

Fig. 4. A comparison of non-fatigue to fatigue state in the sEMG signal of the gastrocnemius is shown. Non-fatigue, or the first $1000 \mathrm{~ms}$, is shown on the left and fatigue, or the final $1000 \mathrm{~ms}$, is shown on the right.

Table 1. RMS Percent Change from Non-fatigue to Fatigue Averaged across All Trials. P Values Are Placed Below the Percent Change

\begin{tabular}{|l|l|l|l|}
\hline & Right Leg & Left Leg & Average \\
\hline Lateral Gastrocnemius & $22.02 \%$ & $13.02 \%$ & $17.51 \%$ \\
\hline P Value & 0.295 & 0.109 & \\
\hline Medial Gastrocnemius & $18.60 \%$ & $14.84 \%$ & $16.72 \%$ \\
\hline P Value & 0.338 & 0.018 & \\
\hline Average & $20.31 \%$ & $13.93 \%$ & $17.12 \%$ \\
\hline
\end{tabular}

Fig. 5a shows the scatterplot of the proposed co-contraction based feature extracted from the left leg. It is seen that the values range from 0.025 to 0.18 with one outlier. In most cases, fatigue has a higher value. The statistical test performed on this feature resulted in a P value of 0.04 In Fig. $5 \mathrm{~b}$, the scatterplot of the right leg is shown. The values range from 0.013 to 0.15 with no outliers. Similar to the previous result, fatigue has a higher value in a majority of cases. In this case, the $P$ value of 0.001 is obtained. The scatter in the right leg is less than that of the left leg. This implies intersubject variability is less in the case of right leg. In comparison with the conventional feature, the proposed feature has a better P value. It may be due to the taking into account of the contributions of both the muscles to represent muscle fatigue. 


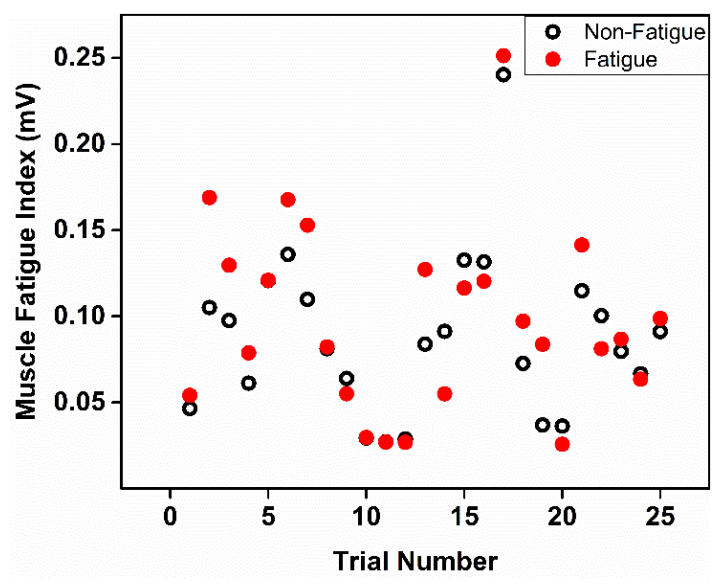

(a) Left Leg

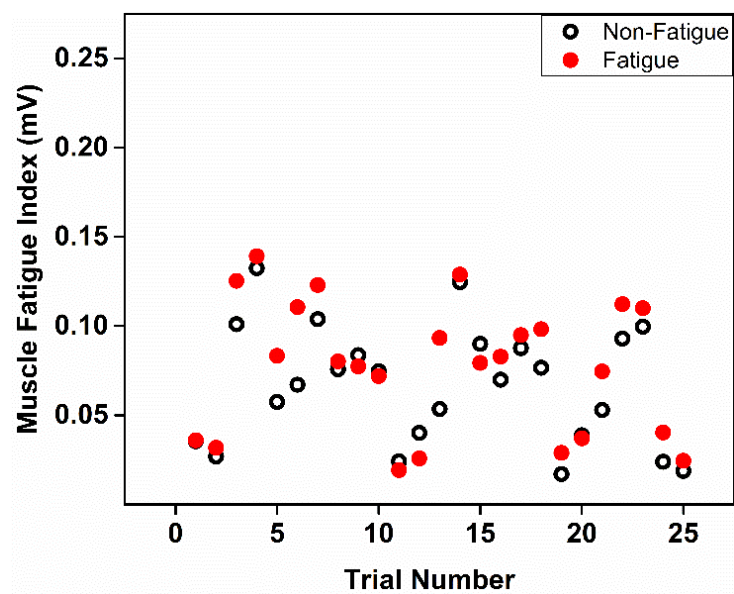

(b) Right Leg

Fig. 5. Non-fatigue and fatigue muscle fatigue index comparison. The proposed feature is able to correctly identify higher values in the fatigue case across almost all cases.

\section{Conclusion}

The purpose of this study is to analyze the variation in the sEMG characteristics during isometric plantar flexion. For this, signals are recorded from the medial and lateral gastrocnemius muscle from 8 subjects leading to a total of 25 trials per leg. The subjects performed isometric plantar flexion and are asked to hold the position until fatigue.

The result shows that the endurance time of each subject vary. On comparing the endurance between legs, a marginal difference is observed. The recorded signal is nonstationary in nature. On visual inspection, the signals show an increase in amplitude with fatigue.

The features such as RMS show an increase in value with $20.311 \%$ in right leg and $13.930 \%$ in the left leg for the signals from medial and lateral gastrocnemius. The proposed muscle fatigue index is a co-contraction based feature and provides better separation between non-fatigue and fatigue. The P-values obtained indicate that the proposed feature performs better than the conventional RMS value with a significance level of $\mathrm{P}<0.01$. This study can be extended to analyze other neuromuscular conditions.

Conclusion

A conclusion section is not required. Although a conclusion may review the main points of the paper, do not replicate the abstract as the conclusion. A conclusion might elaborate on the importance of the work or suggest applications and extensions.

\section{References}

[1] Nordlund, M. M. (2003). Central and peripheral contributions to fatigue in relation to level of activation during repeated maximal voluntary isometric plantar flexions. Journal of Applied Physiology, 96(1) 218-225.

[2] Chowdhury, R. H., et al. (2014). Determination of muscle fatigue in SEMG signal using empirical mode decomposition. IEEE Conference on Biomedical Engineering and Sciences (IECBES), 932-937.

[3] Venugopal, G., et al. (2014). Extraction and analysis of multiple time window features associated with muscle fatigue conditions using sEMG signals. Expert Systems with Applications, 41(6), 2652-2659.

[4] Thrasher, A., et al. (2005). Reducing muscle fatigue due to functional electrical stimulation using random modulation of stimulation parameters. Artificial Organs, 29(6), 453-458.

[5] Giuliom, I. D., et al. (2009). The proprioceptive and agonist roles of gastrocnemius, soleus and tibialis anterior muscles in maintaining human upright posture. The Journal of Physiology, 587(10), 
2399-2416.

[6] Kumar D. K., et al. (2011). Measuring increase in synchronization to identify muscle endurance limit. IEEE Transactions on Neural Systems and Rehabilitation Engineering, 19(5), 578-587.

[7] Merletti, R., \& Parker, P. A. (2010). Electromyography: Physiology, Engineering and Noninvasive Applications. Hoboken, NJ: Wiley-Interscience.

[8] Karthick, P. A., \& Ramakrishnan, S. (2015). Muscle fatigue analysis using surface EMG signals and time-frequency based medium-to-low band power ratio. Electronic Letters, 52(3), 185-186

[9] Young, G. 0. (1964). Synthetic structure of industrial plastics. Plastics (2nd ed.). New York: McGraw-Hill.

[10] Dimitrova, N., \& Dimitrov, G. (2003). Interpretation of EMG changes with fatigue: Facts, pitfalls, and fallacies. Journal of Electromyography and Kinesiology, 13(1), 13-36.

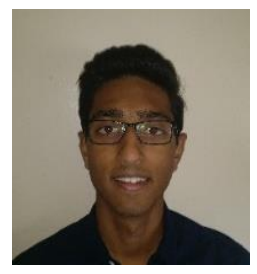

Mayank Patel was born in Lansdale, Pennsylvania in 1998. He is working towards a B.S. degree in biomedical engineering at Drexel University and is enrolled in a BS/MD dual degree program so will receive an M.D. from Drexel University College of Medicine. He is currently working as an intern at the Non-Invasive Imaging and Diagnostics Lab at IIT Madras in Tamil Nadu, India.

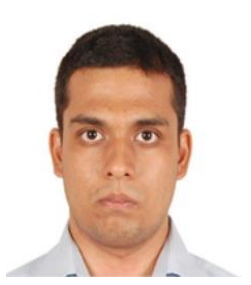

Navaneethkrishna Makaram has a B.E. degree in electrical engineering from Anna University and an M.S. degree in biomedical engineering at the Department of Applied Mechanics, Indian Institute of Technology Madras. He is currently pursuing his Ph.D. in the same department. His research interests include biomedical instrumentation, signal processing, and machine learning processing, and machine learning.

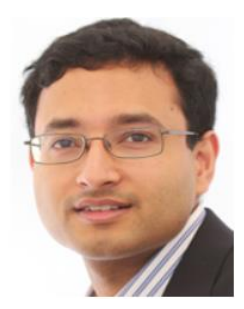

Sriram Balasubramanian was born in Chennai, India. He has a B.S in mechanical engineering, and the M.S in mechanical engineering. He also has a doctorate in biomedical engineering from Wayne State University. His research interests are in orthopedic biomechanics, pediatric spine and rib cage deformities, scoliosis, and computational modeling. He is an associate professor at Drexel University in the School of Biomedical Engineering.

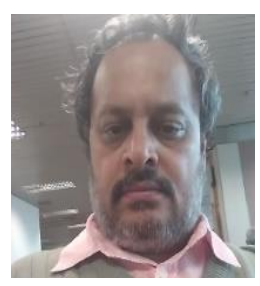

Swaminathan Ramakrishnan is a professor in biomedical engineering at the Indian Institute of Technology Madras. His research interests include biomedical signal and image processing, biomedical instrumentation, and standards and regulations for medical devices. 